\title{
Propuesta de evaluación multidisciplinar del talento de jóvenes futbolistas Proposal for a multidisciplinary assessment of talented football players
}

\author{
*Jaime Serra-Olivares, **, ***Alejandro Prieto-Ayuso, **Juan Carlos Pastor-Vicedo, **Sixto González-Víllora \\ *Universidad Católica de Temuco (Chile), ${ }^{* *}$ Universidad de Castilla-La Mancha (España), ${ }^{* * *}$ Albacete Balompié S.A.D. (España)
}

\begin{abstract}
Resumen. Ante las limitaciones de la perspectiva tradicional de evaluación del talento del futbolista, son necesarios instrumentos de análisis del rendimiento que faciliten la transferencia de la información reportada al entrenamiento y la competición. En este sentido, el enfoque multidisciplinar de evaluación, fundamentado en la ecología dinámica del movimiento, se presenta como una metodología indispensable para el análisis contextualizado de las variables determinantes del rendimiento deportivo. Al respecto, en este trabajo se expone una propuesta de instrumentos diseñados bajo este enfoque. Se describen cuatro herramientas idóneas para: (i) la identificación y selección del joven futbolista con talento, (ii) el análisis del conocimiento táctico, (iii) la evaluación integrada del rendimiento físico y, (iv) el análisis de los procesos de toma de decisión y ejecución técnico-táctica. La utilización de este tipo de procesos evaluativos, permitirá una aproximación de mayor calidad al entendimiento de los factores influyentes sobre el talento y el rendimiento.

Palabras clave: nivel de pericia, evaluación, dinámica ecológica, táctica, deporte.
\end{abstract}

\begin{abstract}
Given the limitations of the traditional perspective of talent assessment in sport, new instruments of performance analysis are necessary to facilitate the transferability to training and competition of the information reported. In this sense, the multidisciplinary approach of assessment, based on the dynamic ecological theories, would be an indispensable methodology for the contextualized analysis of sport performance variables. In this regard, this paper presents a proposal of some instruments designed under this approach. Four tools are described for: (i) talented young players' identification and selection processes, (ii) analysis of tactical knowledge, (iii) integrated assessment of physical performance and, (iv) Decision making and technical-tactical execution evaluation. The use of this kind of assessment instruments and processes will allow a better quality approach to the understanding of the factors that are influencing talent and performance in football.
\end{abstract}

Keywords: expertise, assessment, dynamical ecology, tactics, sport.

\section{Introducción}

El fútbol es uno de los deportes de invasión más practicados en el mundo (Contreras, García-López, y Cervelló, 2005). Por esta razón, es una de las modalidades deportivas más investigadas en años recientes (Brito, Nassis, Seabra y Figueiredo, 2018). La evaluación del rendimiento del futbolista ha sido un tema de gran interés en la última década. Según expertos como Gómez-Ruano (2017), se debe a la existencia de una elevada complejidad e imprevisibilidad de este deporte, que requiere procesos observacionales rigurosos para la comprensión de este fenómeno. Además, constituye un tema trascendental en el área de investigación y también en el ámbito del fútbol de élite (Pankhurst y Collins, 2013) y de aquellas que pretenden llegar a ese nivel (Chamorro, Oliva, González y Alonso, 2016). Por estos motivos, se precisa de un consenso, al menos en cada una de estas parcelas del conocimiento, en relación a la fiabilidad, validez y transferencia de las técnicas e instrumentos empleados (Nicolairé, Catuzzo y de Mello Monteiro, 2013).

La corriente actual sobre el análisis del rendimiento está basada en modelos de análisis del entrenamiento y la competición (en jugadores de formación o alto rendimiento, entrenadores, árbitros u otros roles), y no en situaciones aisladas de laboratorio (Serra-Olivares, Clemente y GonzálezVíllora, 2016). Este proceso permite una mayor contextualización de la evaluación, y favorece la transferencia de tales procedimientos a la investigación aplicada (Memmert, 2010). El análisis contextualizado del talento del jugador para tomar decisiones y ejecutar habilidades técni-

Fecha recepción: 25-06-19. Fecha de aceptación: 20-10-19

Alejandro Prieto-Ayuso

Alejandro.Prieto@uclm.es co-tácticas, por ejemplo, se torna fundamental para el desarrollo óptimo de procesos de formación (González-Víllora, García-López y Contreras-Jordán, 2015; Serra-Olivares, González-Víllora, García-López y Araújo, 2015). En los deportes de invasión como el fútbol, en los cuales se juega en un espacio común y acción simultánea, este factor posee si cabe mayor importancia. Esto se debe a la necesidad de evaluar las variables del talento determinantes sobre el rendimiento en situaciones reales, o en su defecto, en situaciones simuladas (Araújo, 2009; Davids, Button, Araújo, Renshaw y Hristovski, 2006). De este modo se asegura un análisis adaptado a la naturaleza común y específica de los comportamientos de juego.

Algunos ejemplos de la evaluación de otros factores influyentes del rendimiento desde este enfoque son: la valoración de las variables determinantes del talento (Prieto-Ayuso, Pastor-Vicedo y Contreras-Jordán, 2017), el análisis del conocimiento táctico aplicado (Delgado, Longoria, Enríquez, Luján y Cuadras, 2019; Serra-Olivares y García-López, 2016), o la evaluación integrada del rendimiento físico (Lidor, Coté y Hackfort, 2009; Rodriguez, Montenegro y Petro, 2019). Estos procedimientos se alejan de enfoques tradicionales más focalizados en la evaluación de la técnica y la biomecánica de las acciones, facilitando un análisis comportamental contextualizado. Se consigue de este modo información más rigurosa que favorece una mayor trasferencia de los resultados a los procesos de entrenamiento y competición (Clemente, Martins, Kalamaras, Wong y Mendes, 2015).

Al respecto, este trabajo tiene como propósito la exposición de una propuesta de análisis desde el enfoque de los sistemas dinámicos, la psicología ecológica y la perspectiva de los condicionantes del aprendizaje motor (Araújo, 2009; Davids et al., 2008, Newel, 1986). Con este objetivo: (1) se 
presenta una revisión de las variables del talento determinantes del rendimiento del futbolista, (2) se refuerza la necesidad de la evaluación contextualizada del rendimiento en etapas de iniciación y, (3) se expone una propuesta de evaluación multidisciplinar del talento del joven futbolista mediante la utilización de instrumentos de análisis del rendimiento contextualizados.

\section{Talento y determinantes del rendimiento en fútbol}

El estudio del talento y las variables influyentes sobre el rendimiento del futbolista ha sido tratado con anterioridad (Fernández-Río y Méndez-Giménez, 2014). A principios de este siglo, Williams y Reilly (2000) concluyeron que las variables antropométricas, fisiológicas, psicológicas y sociológicas, podrían actuar como predictores del rendimiento. Todavía hoy se mantiene dicha hipótesis, aunque con algunos matices. En la actualidad, parece haber un consenso en señalar que los factores perceptivos y de toma de decisiones determinan el rendimiento (González-Víllora y Costa, 2016; Serra-Olivares, Clemente y González-Víllora, 2016; Woods, Raynor, Bruce y McDonald, 2015), junto a otros aspectos como la personalidad (Morley, Morgan, McKenna y Nicholls, 2014), el enfoque mental, la actitud (Saether, 2014), la efectividad del jugador (Barnes, Archer, Hogg, Bush y Bradley, 2014) y las cualidades sociales (Pazo Haro, Sáenz-López Buñuel, Fradua Uriondo, Barata Figueiredo y Coelho e Silva, 2011). Sin olvidarnos de los factores físicos, condicionales y antropométricos, que también han sido estudiados por investigadores, especialmente durante los procesos de formación (Bidaurrazaga-Letona, Lekue, Amado, Santos-Concejero y Maria Gil, 2015). En la tabla 1 se exponen algunos de los indicadores influyentes sobre el rendimiento en fútbol más analizados hasta la actualidad. Como puede observarse, se trata de la evaluación de indicadores aislados, que junto con el análisis de otras parcelas del rendimiento, aportarían información muy valiosa respecto a la formación y el rendimiento del futbolista.

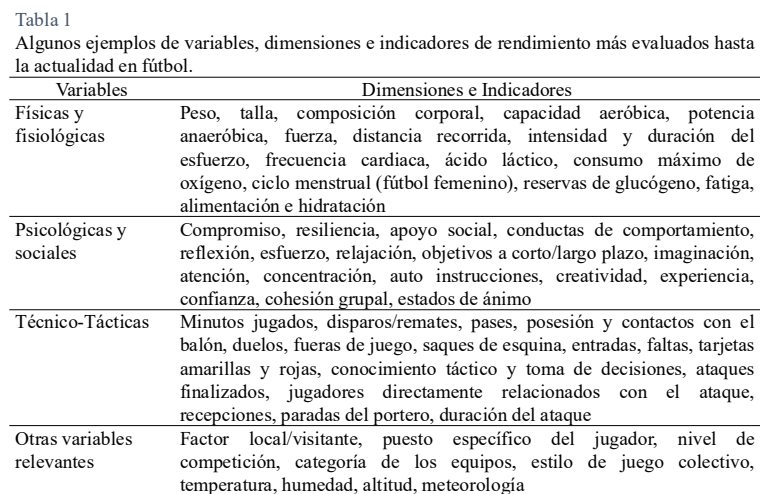

En esta línea, si bien el análisis aislado de indicadores es efectivo durante el desarrollo del método científico, en ocasiones es insuficiente para la evaluación integrada del talento, y su consiguiente desarrollo (García-López et al., 2013). Por esta razón, el diseño representativo de tareas de entrenamiento y evaluación ha adquirido tanta importancia en la actualidad (Serra-Olivares y García-Rubio, 2016; SerraOlivares et al., 2016). Gracias a las aportaciones de las teorías de los sistemas dinámicos, la psicología ecológica, y la pers- pectiva de los condicionantes del aprendizaje motor, actualmente se dispone de un fundamento científico sólido sobre los factores influyentes sobre el talento y el rendimiento deportivo. Desde estas teorías, se considera al deportista como un sistema dinámico flexible que se adapta a los condicionantes impuestos por el contexto, interaccionando mediante procesos de acoplamiento entre información-movimiento. Por su parte, el rendimiento correspondería a la interacción entre los condicionantes individuales del jugador, el entorno y la tarea realizada (Araújo, 2013; Newel, 1986). De modo que, comprendiendo los condicionantes (factores) que afectan al resultado de cada acción deportiva, se conseguirían diseñar procedimientos de análisis más ecológicos (Davids et al., 2013). Con esta idea, el proceso de evaluación del talento y de análisis del rendimiento adquiere un nivel superior de refinamiento (Pinder, Davids, Renshaw y Araújo, 2011; Travassos, Duarte, Vilar, Davids y Araújo, 2012).

\section{Hacia un enfoque integrado de la evaluación del rendi- miento}

Los deportistas toman decisiones y ejecutan acciones utilizando estructuras psicológicas, de conocimiento previo y habilidades de percepción, para adaptarse al contexto mediante procesos de acoplamiento entre información-movimiento (Araújo, 2009; Starkes y Ericsson, 2003). Así, una buena base cognitivo-social-física, junto al entrenamiento perceptivo-decisional, favorecen el desarrollo de comportamientos de juego más eficientes (Davids et al., 2013; Travassos et al., 2012). Por estos motivos, el interés por la evaluación de variables como las características del talento, el conocimiento previo, el rendimiento físico y las habilidades de toma de decisión, dentro de los procesos de evaluación y planificación del entrenamiento del joven futbolista (Serra-Olivares y García-Rubio, 2016).

Sin embargo, en el caso de las características psicológicas, se han utilizado técnicas e instrumentos de evaluación poco funcionales (Morley, Morgan, McKenna y Nicholls, 2014). Resulta una paradoja que, pese a la importancia de los procesos de detección dentro del ámbito del fútbol, no exista una herramienta que permita desarrollar un proceso válido, transferible y de calidad de la evaluación del rendimiento (Nicolairé et al., 2013). Además, la escasa fiabilidad demostrada de los indicadores analizados (Prieto-Ayuso et al., 2017), junto al uso de test y pruebas descontextualizadas, han puesto en duda la utilización de estos procedimientos (Lidor, Coté y Hackfort, 2009).

En relación al análisis del conocimiento y su relación con el rendimiento ha ocurrido algo similar. Si bien el enfoque tradicional posee un fundamento sólido (Starkes y Ericcson, 2003; Ward y Williams, 2003), las herramientas empleadas se han limitado a la evaluación de taxonomías que tienen poca transferencia sobre el entrenamiento y el aprendizaje (Blomqvist, Vänttinen y Luhtanen, 2005). Por otro lado, existen técnicas de análisis que poseen limitaciones metodológicas respecto a otras (González-Víllora, GarcíaLópez, Pastor y Contreras, 2010). Por este motivo, se precisa de instrumentos de evaluación acordes a la realidad de juego (Serra-Olivares y García-López, 2015).

En el caso del análisis del rendimiento en las variables 
físicas y fisiológicas, si bien se percibe una transición, es necesario un ajuste. Tradicionalmente, se han empleado test de evaluación de los indicadores de fuerza, velocidad y resistencia propios de los deportes intermitentes como el fútbol (Prieto-Ayuso et al., 2017), pero dichas pruebas no parecen estar claramente definidas, y son ambiguas (Nicolairé et al., 2013). Pese a la existencia de multitud de ejemplos, carecen de fiabilidad, y se observan discrepancias para medir una misma variable. En este sentido, dada la naturaleza dinámica y condicional del deporte del fútbol (Randers, Andersen, Rasmussen, Larsen y Krustrup, 2014), se precisa de una perspectiva de análisis más innovadora y centrada en los requerimientos condicionales específicos. Así, gracias a la aplicación de nuevas técnicas como los dispositivos de monitorización (video, pulsómetros o GPS) (Núñez, ToscanoBendala, Suárez-Arrones, Martínez-Cabrera y De Hoyo, 2019), se puede obtener una gran cantidad de datos contextualizados (Ballesta Castells et al., 2015; Liu et al., 2015). Sin embargo, la transferencia de tal información no ha sido del todo extendida desde la investigación al entrenamiento y la competición, sobretodo en etapas formativas (Nicolaire et al., 2016; Rebelo, Brito, Seabra, Oliveira y Krustrup, 2014). Por este motivo, se precisa del diseño de pruebas en contextos reales de juego, que permitan una evaluación eficiente del rendimiento en las variables físicas y fisiológicas.

Finalmente, en la evaluación de las habilidades técnicotácticas se observa el mismo problema. Las pruebas realizadas se han orientado a la evaluación de destrezas técnicas que carecen del componente perceptivo-decisional de juego en fútbol (Serra-Olivares et al., 2016). Por otro lado, la identificación y selección de jóvenes futbolistas sigue realizándose en base a la capacidad de entrenadores y ojeadores para determinar las habilidades del jugador en situaciones de competición. Incluso a veces, en estas pruebas el jugador no interacciona con el balón. Por este motivo, se han construido instrumentos de análisis de la toma de decisiones y ejecución técnico-táctica en situación de juego. Sin embargo, si bien el nuevo enfoque ha supuesto un avance para el conocimiento científico, se ha limitado a la evaluación de la toma de decisiones comportamental, sin atender al contexto táctico de las situaciones (González-Víllora et al., 2015). Este aspecto subraya la necesidad de diseñar herramientas más ecológicas (García López et al., 2013).

Con ello, a pesar de que los procedimientos de evaluación conocidos permiten el análisis de factores influyentes sobre el talento, poseen limitaciones para proporcionar una visión multifacética del rendimiento. De tal manera que, una perspectiva de análisis multidisciplinar y contextualizada, facilitaría la aplicabilidad y transferencia de la información resultante a los procesos de formación. Este es un factor importante para el desarrollo del talento que debe ser tenido en cuenta tanto por investigadores, como por los profesionales encargados del desarrollo del joven futbolista. En este sentido, en la tabla 2 se exponen algunos ejemplos de instrumentos de evaluación que poseen una visión más integradora. Están fundamentados en el análisis del rendimiento desde un enfoque más integral y flexible, adaptado a la realidad dinámica del rendimiento. Se trata de un enfoque más ecológico, dado que se analizan las variables teniendo presente la relación jugador/contexto, y la interacción de los condicionantes que afectan al rendimiento.

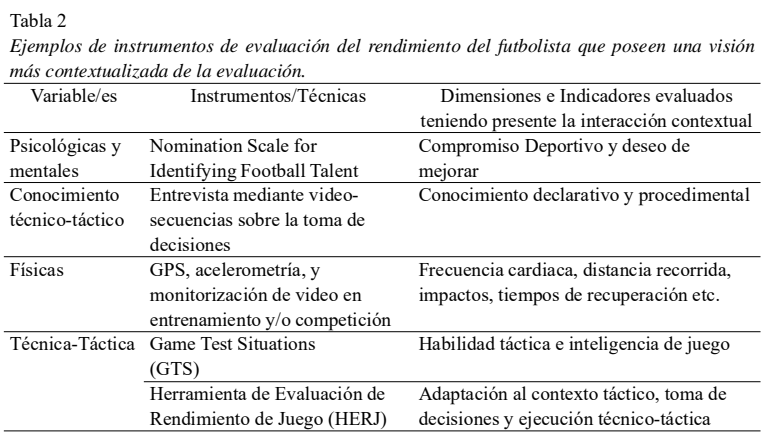

Con un enfoque multidisciplinar e integrado, se aborda un análisis multidimensional del juego o el rendimiento, el cual aporta información relevante sobre indicadores físicos/ fisiológicos, y también técnicos-tácticos, estratégicos y psicosociales. Se adopta así una perspectiva evaluativa más completa sobre la complejidad y variabilidad del rendimiento (Passos, Araújo y Volossovitch, 2016; Gómez-Ruano, 2017). En este sentido, a continuación se expone una propuesta de evaluación del talento mediante la utilización de cuatro instrumentos fundamentados en esta perspectiva, y que pueden emplearse conjuntamente para la identificación, selección y formación del joven futbolista.

\section{Propuesta de evaluación multidisciplinar contextualizada del joven futbolista}

Evaluación integrada del joven futbolista con talento: Nomination Scale for Identifying Football Talent (NSIFT)

La escala NSIFT, a diferencia de otros procesos de identificación y selección de jóvenes talentos basados en la intuición del entrenador, permite la evaluación multifacética del rendimiento del joven futbolista en los factores cognitivos del juego, psicológicos y motivacionales. Los únicos antecedentes de este tipo de procesos, parten del ámbito educativo, advirtiendo que la triangulación de la información derivada del profesorado, padres y compañeros, otorga una gran fiabilidad a la hora de determinar quiénes son los alumnos (en este caso futbolistas) con altas capacidades intelectuales (Renzulli y Gaesser, 2015).

El diseño se basa en una adaptación de los procesos de detección realizados hasta la actualidad en el ámbito educativo, a la evaluación del rendimiento del joven jugador de fútbol (Prieto-Ayuso et al., 2017). Así, el instrumento tiene en cuenta la opinión del experto (entrenador) y, además, la evaluación global del jugador a través de la opinión de padres y compañeros. Este hecho permite la obtención de valiosa información sobre la realidad del jugador que, con la utilización de otras herramientas, pasaría desapercibida.

La NSIFT ha sido validada en contenido por 16 expertos, ocho de ellos pertenecientes al área de Ciencias de la Actividad Física y el Deporte, y ocho entrenadores de fútbol con el nivel III, en activo y pertenecientes a clubs profesionales de fútbol. Ambos con un mínimo de 10 años de experiencia en este campo del conocimiento. El instrumento ha superado el procedimiento de análisis factorial exploratorio. El estudio piloto con 556 jugadores de fútbol pertenecientes a categorías de formación (sub-10 a sub-23) de un club de fútbol 
profesional español, ha permitido afirmar que se trata de una herramienta adecuada para la evaluación del talento previo del joven futbolista en categorías inferiores de fútbol. En la tabla 3 , se exponen las variables, dimensiones e indicadores evaluados mediante la NSIFT.

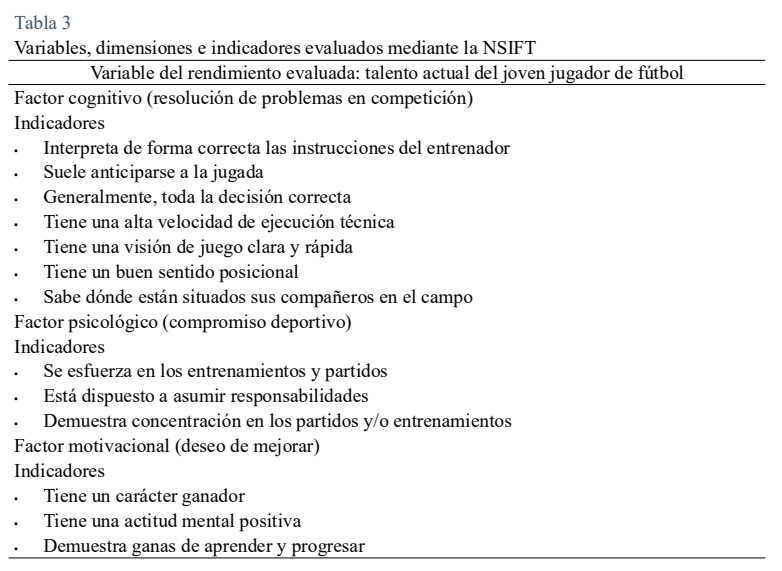

Evaluación del conocimiento aplicado: EI Test de Conocimiento Táctico (TCTOF)

El TCTOF es un instrumento fundamentado en los paradigmas del conocimiento y del rendimiento experto, en las teorías de la psicología ecológica y los sistemas dinámico, y en la perspectiva de los condicionantes del aprendizaje motor (Newell, 1986). Estas corrientes explican el rendimiento en el deporte asumiendo la existencia de factores perceptivo-cognitivos que facilitan la utilización de un conocimiento flexible, funcional y adaptable en el deporte. En este sentido, el TCTOF se diferencia del resto de herramientas en que se contextualizan los ítems en relación a la dinámica de juego y la problemática táctica (Serra-Olivares y GarcíaRubio, 2017).

Así, se evalúa el conocimiento táctico del joven futbolista en sus dimensiones declarativa (saber qué y para qué hacer) y procedimental (saber cómo, por qué y cuándo hacer), de acuerdo a la interacción espacio-temporal e individual/colectiva propia del juego. En la tabla 4, se presenta la estructura del TCTOF respecto a la variable, dimensiones e indicadores del rendimiento cognitivo que se evalúan.

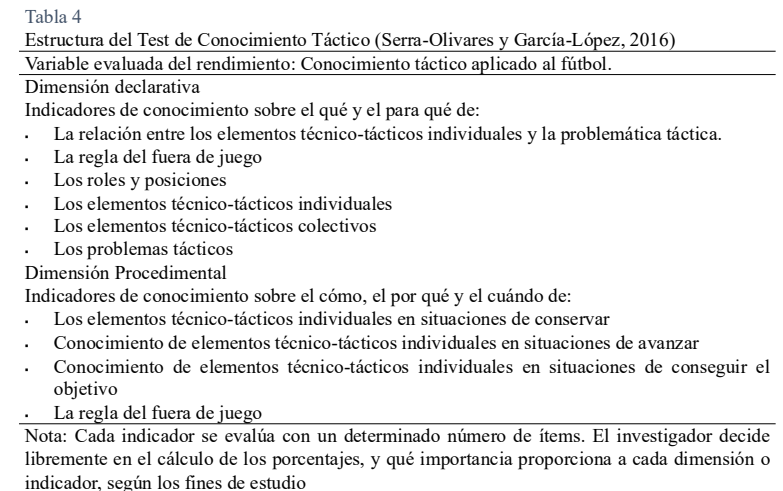

El test presenta resultados adecuados de fiabilidad y validez (Serra-Olivares y García-López, 2015), y ha sido utilizado para analizar el conocimiento sobre la táctica en el contexto de iniciación al deporte (Serra-Olivares, 2014; SerraOlivares, García-López y Gutiérrez-Díaz del campo, 2017), las taxonomías relacionadas con la táctica en jóvenes talentos en canteras de fútbol profesional (Serra-Olivares et al., 2015a), y la relación entre el conocimiento táctico, la experiencia y el nivel de pericia de jóvenes futbolistas (Serra-Olivares et al., 2015b). Por todo lo anterior, se sugiere su utilización para el análisis del rendimiento cognitivo del joven jugador de fútbol en relación a la dinámica contextual del juego, de una forma sencilla y económica.

Evaluación del rendimiento físico y fisiológico: Análisis contextualizado de las variables condicionales a través de dispositivos de monitorización

El interés por conocer las exigencias físicas del futbolista con el propósito de mejorar su rendimiento no es una novedad, y ha conllevado la realización de interesantes estudios (Casamichana, Castellano y Castagna, 2012; Gutierrez, Paulis, Gómez y Sánchez, 2018) Loturco et al., 2019; Murr, Raabe y Höner, 2018; Serpiello et al., 2018; Stølen, Chamari, Castagna y Wisløff, 2005; Trecroci, Milanoviæ, Frontini, Iaia yAlberti, 2018). Hasta la fecha, se han propuesto diferentes metodologías de evaluación del rendimiento del futbolista profesional, y otros instrumentos orientados a la evaluación del talento en etapas de formación respecto a las variables físicas y fisiológicas. Sin embargo, se observan discrepancias en a las estrategias de utilización, y diferencias en cuanto a la precisión para analizar dimensiones e indicadores relacionados con el rendimiento condicional.

Con ello, se percibe la necesidad de construir herramientas de evaluación contextualizada del rendimiento en las variables físicas y fisiológicas, que contribuyan a una mejor comprensión del talento y el rendimiento deportivo. Este aspecto es aún más importante si se trata de la evaluación de tales variables en etapas de formación, en las que el conocimiento sobre los factores que influyen sobre el rendimiento es fundamental para la planificación de procesos formativos eficientes.

Si bien es cierto que se han desarrollado estudios que analizaron las exigencias físicas del joven futbolista en situaciones contextualizadas como los juegos modificados (Aasgaard y Kilding, 2018; Asian-Clemente, Sáez de Villarreal, Nayler y Requena, 2019; Bujalance-Moreno, Latorre-Román y García-Pinillos, 2019; Castellano, Echeazarra y Estéfano, 2017; Castellano y Echeazarra, 2013; Coutinho, Gonçalves, Santos, Travassos, Wong y Sampaio, 2018; Pascual, Orbea, Martinez y Jove et al., 2015; Práxedes, Moreno, Gil-Arias, Claver y Del Villar, 2018; Rábano-Muñoz, Asian-Clemente, Sáez de Villarreal, Nayler y Requena, 2019; Sánchez-Sánchez, García, Asián-Clemente, Nakamura y Ramírez-Campillo, in press), se alerta de la necesidad de completar dicha información con situaciones de análisis en competición (Casamichana, Castellano y Castagna., 2012). Este proceso, aportaría la información necesaria para la planificación de tareas condicionales ajustadas a las necesidades de los jugadores y equipos.

En este sentido, la evaluación multidisciplinar del talento del joven futbolista puede ser complementada por medio de la obtención de información a partir del uso de Sistemas de Seguimiento Computarizados (Castellano y Álvarez-Pastor, 2014; Kêsicki y Lewicki, 2017; Mallo et al., 2015). Este tipo de dispositivos no sólo permiten identificar las variables cinemáticas, sino también las fisiológicas, siendo los datos 
obtenidos más fiables que aquellos proporcionados por otras pruebas tradicionales de análisis aislado de indicadores. Así, la información proporcionada por el análisis contextualizado de las variables condicionales puede transferirse fácilmente al diseño de tareas y entrenamientos, orientados a mejorar el rendimiento. Además, como recomiendan algunos trabajos (Casamichana y Castellano, 2010; Pascual et al., 2015; Castellano, 2017), el empleo de estos dispositivos puede ser utilizado con éxito para identificar las demandas cinemáticas y fisiológicas. Estos procesos pueden ser desarrollados con el propósito de contribuir a la mejora de tales variables durante el proceso formativo, y también, como medio de identificación y desarrollo del talento en esta parcela del rendimiento.

Al respecto, se presenta una propuesta de análisis del rendimiento físico y fisiológico en el futbol de formación mediante la utilización de dispositivos de monitorización en situaciones de entrenamiento y competición. Este método puede ser combinado con otras herramientas de evaluación del talento como las expuestas anteriormente. De este modo, se consigue obtener una panorámica ajustada a la realidad del futbolista en esta etapa, evitando así cualquier sesgo subjetivo, como por ejemplo, el Efecto de la edad Relativa (Bidaurrazaga-Letona et al., 2014).

En este caso, el instrumento expuesto corresponde al dispositivo de monitorización WIMU (Molina-Carmona, Gomez-Carmona, Bastida Castillo y Pino-Ortega, 2018; Muñoz-López, Granero-Gil, Pino-Ortega y De Hoyo, 2017), de Real Track Systems, y al Software Qüiqo. Esta herramienta ha sido utilizada previamente con jóvenes futbolistas, y proporciona datos fiables y válidos respecto a las variables determinantes del rendimiento condicional (Pastor-Vicedo, González-Víllora, Serra-Olivares y Hernández-Martínez, 2014). Mediante el uso de este dispositivo y las terminales integradas (acelerometría, giroscopio, frecuencia cardiaca y otros vectores de movimiento), se puede obtener información relevante sobre variables locomotoras, metabólicas y mecánicas, esenciales para un adecuado seguimiento y control del rendimiento de los deportistas. En la figura 2, se presenta un ejemplo de información proporcionada por el dispositivo

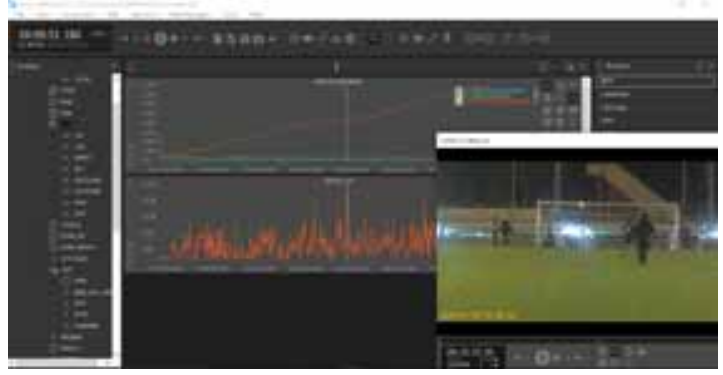

Figura 1. Ejemplo de un momento de análisis contextualizado del rendimiento de jóvenes futbolistas en variables fisicas y fisiológicas.

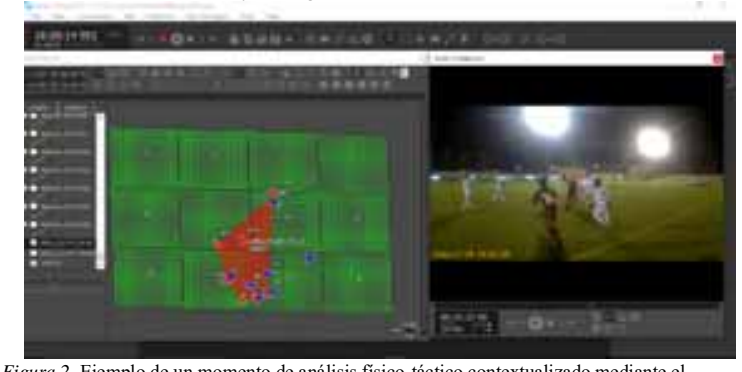

Figura 2. Ejemplo de un momento de análisis físico-táctico contextualizado mediante el dispositivo WIMU. durante el análisis físico y fisiológico de jóvenes futbolistas en situaciones de F7. Igualmente, en la figura 3 se expone un ejemplo de un momento de análisis condicional, complementario al análisis táctico de la situación de juego.

En definitiva, mediante este tipo de dispositivos se pueden monitorizar de manera contextualizada una gran cantidad de variables, dimensiones e indicadores relacionados con las exigencias físicas y fisiológicas de los jugadores. Con ello, se proporciona información relevante y complementaria respeto a la evaluación del talento y el análisis del rendimiento. Este tipo de información permite la transferencia de los hallazgos observados a los procesos de formación y desarrollo del joven futbolista.

Evaluación de la toma de decisiones y la ejecución técnico-táctica: El Game Performance Evaluation Tool (GPET)

El instrumento GPET (García-López, González-Víllora, Gutiérrez-Díaz y Serra-Olivares, 2013), ha sido diseñado para la evaluación del rendimiento de juego (toma de decisiones y ejecución técnico-táctica) del joven iniciado a los deportes de invasión como el fútbol. Ofrece la posibilidad de analizar cada decisión de juego desde el punto de vista del problema táctico que el jugador tiene que resolver en cada situación.

Este enfoque, permite una evaluación más ecológica de la toma de decisiones que aquella adoptada en anteriores herramientas en los que se analizó la toma de decisiones y la ejecución de la habilidad, sin considerar la problemática táctica de las situaciones de juego (Serra-Olivares, GonzálezVíllora, García-Lópezy Araújo, 2015; Serra-Olivares, GonzálezVíllora y García-López, 2015). En la tabla 5 se expone la variable, dimensiones e indicadores del rendimiento evaluados mediante el GPET.

Mediante el GPET se evalúa el rendimiento del juego en dos niveles. En el primer nivel, se evalúa la adaptación de las acciones a los problemas tácticos en ataque: 1) conservar el balón, 2) progresar hacia la portería contraria y 3) conseguir gol. En el segundo nivel, se evalúan los componentes cognitivos de la toma de decisiones y los componentes de la habilidad motriz de la ejecución de manera contextualizada.

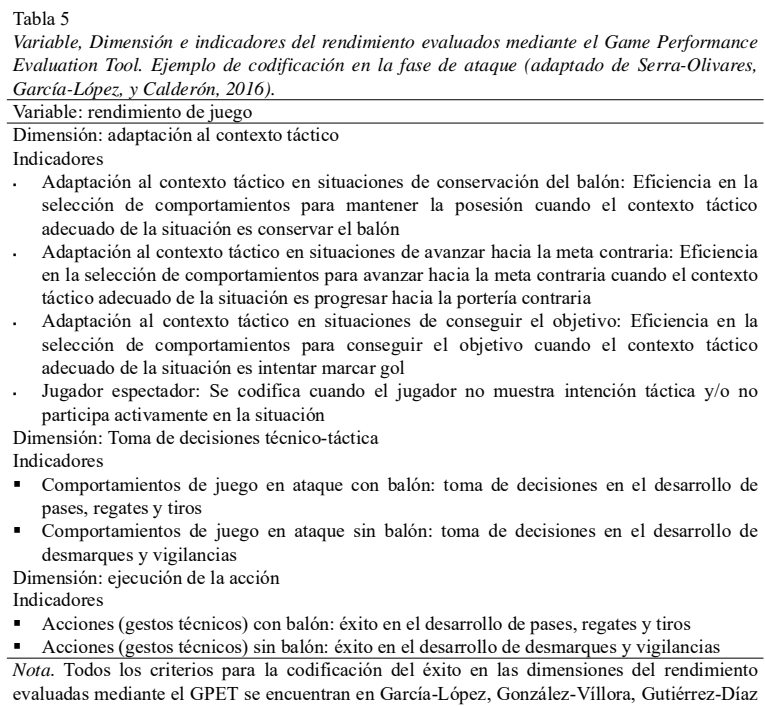

Variable, Dimensión e indicadores del rendimiento evaluados mediante el Game Performance Evaluation Tool. Ejemplo de codificación en la fase de ataque (adaptado de Serra-Olivares, García-López, y Calderón, 2016).

Variable: rendimiento de juego

Dimensión: adaptación al contexto táctico

Indicadores

Adaptación al contexto táctico en situaciones de conservación del balón: Eficiencia en la selección de comportamientos para mantener la posesión cuando el contexto táctico adecuado de la situación es conservar el balón

Adaptación al contexto táctico en situaciones de avanzar hacia la meta contraria: Eficiencia en la selección de comportamientos para avanzar hacia la meta contraria cuando el contexto táctico adecuado de la situación es progresar hacia la portería contraria

Adaptación al contexto táctico en situaciones de conseguir el objetivo: Eficiencia en la selección de comportamientos para conseguir el objetivo cuando el contexto táctico adecuado de la situación es intentar marcar gol

Jugador espectador: Se codifica cuando el jugador no muestra intención táctica y/o no participa activamente en la situación

Dimensión: Toma de decisiones técnico-táctica

Indicadores

Comportamientos de juego en ataque con balón: toma de decisiones en el desarrollo de pases, regates y tiros

Comportamientos de juego en ataque sin balón: toma de decisiones en el desarrollo de desmarques y vigilancias

Dimensión: ejecución de la acción

Indicadores

- Acciones (gestos técnicos) con balón: éxito en el desarrollo de pases, regates y tiros - Acciones (gestos técnicos) sin balón: éxito en el desarrollo de desmarques y vigilancias

Nota. Todos los criterios para la codificación del éxito en las dimensiones del rendimiento evaluadas mediante el GPET se encuentran en García-López, González-Víllora, Gutiérrez-Díaz y Serra-Olivares (2013) 
El GPET se puede utilizar en el ámbito educativo y también el deportivo, con fines de estudio o de aplicabilidad al entrenamiento y la competición (Serra-Olivares et al., 2016). Es ideal para la evaluación de la adaptación del joven jugador al contexto de juego, siendo ideal su aplicación en la fase de la iniciación deportiva (6-14 años), según los diferentes ritmos de aprendizaje. No obstante, puede emplearse en adultos, junto a la utilización de otras técnicas de evaluación del rendimiento respecto a la táctica deportiva (herramientas de evaluación del conocimiento táctico, análisis táctico mediante video, dispositivos GPS).

El GPET ha sido validado previamente y utilizado en varios estudios (González-Víllora, García-López y ContrerasJordán, 2015; González-Víllora, García-López, ContrerasJordán y Gutiérrez-Díaz, 2010; González-Víllora, García-López, Pastor-Vicedo y Contreras-Jordán, 2011; Serra-Olivares et al., 2015; Serra-Olivares et al., 2016). Presenta resultados satisfactorios en su fiabilidad inter e intra-observador. Igualmente, posee una consistencia interna adecuada, y se ha adecuado a la validez de criterio de evaluación del rendimiento de otros instrumentos o técnicas como la evaluación del talento por parte de entrenadores o el análisis del conocimiento táctico mediante test escritos.

Así, el GPET responde al objetivo esencial de la evaluación deportiva como formación continua del jugador, favoreciendo el énfasis en el desarrollo de jugadores capaces de controlar el mayor número de variables técnico-tácticas posibles, en un tiempo reducido y eligiendo la mejor opción en cada momento de juego (González-Víllora et al., 2015). Por tanto, favorece la evaluación formativa de jugadores autónomos y que no sean dependientes de sus entrenadores/ profesores, en especial en la toma de decisiones. Esto permitiría desarrollar jugadores inteligentes y creativos (Memmert, 2010)

\section{Conclusiones}

A pesar de las aportaciones de la perspectiva tradicional de evaluación del talento en deportes como el fútbol, se requiere un cambio en los procedimientos de análisis del rendimiento. Al respecto, el estado actual del conocimiento científico sobre las variables determinantes y la naturaleza de los comportamientos de juego efectivos, subrayan la necesidad de adoptar un enfoque multidisciplinar e integrado. Este enfoque permitirá una aproximación de mayor calidad al entendimiento de los factores influyentes sobre el rendimiento. Y también, facilitará la transferencia del conocimiento científico al entrenamiento y la competición, principalmente en las etapas de iniciación del joven futbolista. En este sentido, la utilización combinada de pruebas y test contextualizados como los expuestos en este trabajo (NSIFT, TCTOF, WIMU, y GPET), se sugiere idónea en la transición al cambio de enfoque en el análisis del rendimiento. En todo caso, el talento y el rendimiento en deportes como el fútbol son constructos complejos que engloban la interacción de multitud de variables, psicológicas, sociales, físicas y técnico-tácticas. Por este motivo, se precisa de más trabajos de investigación aplicada que analicen la trasferencia de la información obtenida a través del método científico, al ámbito profesional de la elite en el deporte.

\section{Aplicaciones prácticas y prospectiva de estudio}

El enfoque multidisciplinar de evaluación del rendimiento, a través de la utilización de instrumentos de análisis de corte ecológico como los de este trabajo, se sugiere interesante y necesario. La aplicación de este tipo de herramientas ha sido empleada con anterioridad en academias de equipos de fútbol profesional (González-Víllora et al., 2010; PrietoAyuso et al., 2017; Serra-Olivares et al., 2015; Serra-Olivares et al., 2016), y proporciona información fácilmente utilizable por los entrenadores, preparadores físicos y encargados de la formación del joven futbolista, y con transferencia al entrenamiento y competición. El hecho de comprender las variables que influyen sobre el talento desde los diferentes actores (familiares, compañeros, cuerpo técnico), permitirá un abordaje óptimo de los procesos de identificación y selección del talento. Igualmente, entender cómo estructuran y utilizan los jugadores el conocimiento previo facilitará la organización de procesos de enseñanza-entrenamiento adaptados a sus características. De igual modo, conocer las necesidades locomotoras, metabólicas y mecánicas de los jugadores y las especialidades en función de las posiciones y particularidades del juego, proporcionará datos realmente relevantes para la planificación condicional. Por último, la evaluación del rendimiento técnico-táctico de manera contextualizada conllevará una mejor comprensión de los comportamientos de juego y el talento. Este procedimiento, serviría para la identificación de aquellos indicadores y dimensiones del rendimiento a los que prestar especial atención en los procesos de desarrollo del talento. En definitiva, con un procedimiento multidisciplinar integrado se lograría un enfoque adecuado a la realidad actual.

\section{Referencias}

Arana, J., Lapresa, D., Anguera, M. T., \& Garzoìn, B. (2013). Adapting football to the child: an application of the logistic regression model in observational methodology. Quality \& Quantity, 47(6), 3473-3480. doi: 10.1007/s11135-012-9734-z

Araújo, D. (2013). The study of decision-making behavior in sport. RICYDE. Revista Internacional de Ciencias del Deporte, 9(31), $1-4$.

Aasgaard, M., \& Kilding, A. E. (2018). Does man marking influence running outputs and intensity during small-sided soccer games? Journal of strength and conditioning research.

Ballesta Castells, C., Garciìa Romero, J., Fernaìndez Garciìa, J. C. \& Alvero Cruz, J. R. (2015). Meitodos actuales de anaìlisis del partido de fuitbol / Current Methods of Soccer Match Analysis. Revista Internacional de Medicina y Ciencias de la Actividad Fiìsica y el Deporte, 15(60) pp. 785-803.

Barnes, C., Archer, D. T., Hogg, B., Bush, M., \& Bradley, P. S. (2014). The evolution of physical and technical performance parameters in the English Premier League. International Journal of Sports Medicine, 35(13), 1095-1100. doi: 10.1055/s-00341375695

Bidaurrazaga-Letona, I., Badiola, A., Granados, C., Lekue, J. A., Amado, M., \& Gil, S. M. (2014). Efecto relativo de la edad en futbol: estudio en un club Vasco profesional. Retos: Nuevas tendencias en Educación Física, Deporte y Recreación, 25, 95 99.

Bidaurrazaga-Letona, I.; Lekue, J. A.; Amado, M.; Santos-Concejero, J., \& Gil, S. M. (2014). Identifying talented young soccer players: conditional, anthropometrical and physiological 
characteristics as predictors of performance. RICYDE. Revista Internacional de Ciencias del Deporte, 11(39), 79-95.

Blomqvist, M.; Vänttinen, T., \& Luhtanen, P. (2005). Assessment of secondary school students' decision-making and game-play ability in soccer. Physical Education and Sport Pedagogy, 10(2), 107-119. doi: 10.1080/17408980500104992.

Brito, J., Nassis, G. P., Seabra, A. T., \& Figueiredo, P. (2018). Top 50 most-cited articles in medicine and science in football. BMJ Open Sport Exerc. Med. 4, 1-8. doi:10.1136/bmjsem-2018000388

Bujalance-Moreno, P., Latorre-Román, P. Á., \& García-Pinillos, F. (2019). A systematic review on small-sided games in football players: Acute and chronic adaptations. Journal of Sports Sciences, 37(8), 921-949.

Casamichana, D., \& Castellano, J. (2010). Time-motion, heart rate, perceptual and motor behaviour demands in small-sides soccer games: Effects of pitch size, Journal of Sports Sciences, 28, 1615-1623. DOI: 10.1080/02640414.2010.521168

Casamichana, D., Castellano, J., \& Castagna, C. (2012). Comparing the physical demands of friendly matches and small-sided games in semiprofessional soccer players. Journal of Strength and Conditioning Re- search, 26(3), 837-843. Doi: 10.1519/ JSC.0b013e31822a61cf.

Castellano, J., \& Alvarez-Pastor, D. (2014). Evaluation of Research Using Computerised Tracking Systems (AmiscoOİ and ProzoneOI) to Analyse Physical Performance in Elite Soccer: A Systematic Review. Sports Medicine, 44, 701-712. doi: 10.1007/s40279-014-0144-3

Castellano, J., \& Echeazarra I. (2013). Adapting model competition in youth football: A comparative study of 5-a side football and 7-a side football in U-9 players. In H. Nunome, B. Drust, y D. Dawson (Eds.), Science and football VII (pp. 311-316). London: Routledge.

Castellano, J., Echeazarra, I., \& Estéfano, I. (2017). Comparacioìn de las demandas fiìsicas en jugadores de fuitbol sub13 y sub14 en un 7:7 disputado en diferentes dimensiones. Cultura, Ciencia y Deporte, 12, 55-65.

Chamorro, J. M. L., Oliva, D. S., González, J. J. P., \& Alonso, D. A. (2016). ¿Jugaré en la élite? Expectativas de llegar a profesional y su relación con la motivación en jóvenes jugadores de fútbol. Retos: Nuevas Tendencias en Educación Física, Deporte y Recreación, 29, 153-156.

Clemente, F. M., Martins, F. M., Kalamaras, D., Wong, D. P., \& Mendes, R. S. (2015). General network analysis of national soccer teams in FIFA World Cup 2014. International Journal of Performance Analysis in Sport, 15, 80-96.

Contreras, O. R., García-López, L. M., \& Cervelló, E. (2005). Transfer of tactical knowledge from invasion games to floorball. J. Hum. Mov. Stud., 193-213.

Coutinho, D., Gonçalves, B., Santos, S., Travassos, B., Wong, D. P., \& Sampaio, J. (2018). Effects of the pitch configuration design on players' physical performance and movement behaviour during soccer small-sided games. Research in Sports Medicine, 1-16.

Davids, K., Button, C., Araújo, D., Renshaw, I., \& Hristovski, R. (2006). Movement models from sports provide representative task constraints for studying adaptive behavior in human movement systems. Adaptive Behavior, 14(1), 73-95.

Delgado, J. C., Longoria, R. J., Enríquez, O. N., Luján, R. C., \& Cuadras, G. G. (2019). Sistemas tácticos y resultados de competición del Mundial de Fútbol Asociación de Rusia 2018. Tactical systems and matches results from the Football Association Worldcup Rusia 2018. Retos: Nuevas Tendencias en Educación Física, Deporte y Recreación, 36, 503-509.

Dellal, A., Chamari, K., Wong, D. P., Ahmaidi, S., Keller, D., Barros, R., ... \& Carling, C. (2011). Comparison of physical and technical performance in European soccer match-play: FA Premier League and La Liga. European Journal of Sport Science, 11(1), 51-59.

Fernández-Rio, J., \& Méndez-Giménez,A. (2014). Talent detection and development in soccer: a review. Journal of sport and health research, 6(1), 7-18.

García-López, L. M., González-Víllora, S., Gutiérrez-Díaz, D., \& Serra-Olivares, J. (2013). Development and validation of the game performance evaluation tool (GPET) in soccer. Revista Euroamericana de Ciencias del Deporte, 2(1), 89-99.

Gómez-Ruano, M. Á. (2017). The importance of performance analysis as an emergent research topic in sport sciences. RICYDE. Revista Internacional de Ciencias del Deporte. doi: 10.5232/ricyde, 13(47), 1-4.

González-Víllora, S., \& Costa, I. T. (2016). ¿ How to evaluate the soccer tactics? System of tactical assessment in soccer (FUTSAT). Educación Física y Deporte, 34(2), 467-505.

González-Víllora, S., García-López, L. M., \& Contreras-Jordán, O. R. (2015). Evolución de la toma de decisiones y la habilidad técnica en fútbol. Revista Internacional de Medicina y Ciencias de la Actividad Física y el Deporte, 15(59), 467-487.

González-Víllora, S., García-López, L. M., Contreras-Jordán, O. R., \& Gutiérrez-Díaz del Campo, D. (2010). Estudio descriptivo sobre el desarrollo táctico y la toma de decisiones en jóvenes jugadores de fútbol (12 años). Revista Infancia y Aprendizaje, 33(4), 489-501. doi: 10.1174/021037010793139644.

González-Víllora, S., García-López, L. M., Pastor-Vicedo, J. C., \& Contreras-Jordán, O. R. (2010). Estudio descriptivo sobre el desarrollo táctico y la toma de decisiones en jóvenes jugadores de fútbol (10 años). Revista de Psicología del Deporte, 20(1), 79-97.

González-Víllora, S., Serra-Olivares, J., Pastor-Vicedo, J. C., \& Teoldo, I. (2015). Review of the tactical evaluation tools for youth players, assessing the tactics in team sports: football. SpringerPlus, 4, 663. DOI: 10.1186/s40064-015-1462-0.

Gutierrez, J., Paulis, J. C., Gómez, D. C., \& Sánchez, J. S. (2018). Influencia del tamaño del campo y horario del partido en la respuesta física de equipos de la Segunda División Española de Fútbol. Retos: nuevas tendencias en educación física, deporte y recreación, 33, 213-216.

Kêsicki, G., \& Lewicki, W. (2017). Innovative dimension of using telematics tools in training processes of young football players. Trends in Sport Sciences, 24(4).

Lidor, R., Côté, J., \& Hackfort, D. (2009). ISSP position stand: To test or not to test? The use of physical skill tests in talent detection and in early phases of sport development. International Journal of Sport and Exercise Psychology, 7(2), 131-146

Liu, H., Gómez-Ruano, M. Á., Lago-Peñas, C., \& Sampaio, J. (2015). Match statistics related to winning in the group stage of 2014 Brazil FIFA World Cup. Journal of Sports Sciences, 33(12), 1205-1213.

Loturco, I., Jeffreys, I., Abad, C. C. C., Kobal, R., Zanetti, V., Pereira, L. A, ... \& Nimphius, S. (2019). Change-of-direction, speed and jump performance in soccer players: a comparison across different age-categories. Journal of Sports Sciences, 1-7.

Mallo, J., Mena, E., Nevado, F., \& Paredes, V. (2015). Physical Demands of Top-Class Soccer Friendly Matches in Relation to a Playing Position Using Global Positioning System Technology. Journal of Human Kinetics, 47, 179-188. doi: 10.1515/hukin-2015-0073

Memmert, D. (2010). Testing of tactical performance in youth elite soccer. Journal of Sports Science y Medicine, 9(2), 199205.

Moreno-Domínguez,A., Del Villar, F., García-González, L., GarcíaCalvo, T., \& Moreno, M.P. (2013). Propiedades psicométricas 
de un cuestionario para la evaluación del conocimiento procedimental en voleibol (CCPV). RICYDE, 30(9), 38-47. http://dx.doi.org/10.5232/ricyde2013.03103.

Molina-Carmona, I., Gomez-Carmona, C. D., Bastida Castillo, A., \& Pino-Ortega, J. (2018). Validez del dispositivo inercial WIMU PROTM para el registro de la frecuencia cardíaca en un test de campo. Sportk Revista Euroamericana de Ciencias del Deporte, $7,81-86$.

Morley, D., Morgan, G., McKenna, J., \& Nicholls, A. R. (2014) Developmental contexts and features of elite academy football players: Coach and player perspectives. International journal of Sports Science \& Coaching, 9(1), 217-232.

Muñoz-López, A., Granero-Gil, P., Pino-Ortega, J., \& De Hoyo, M. (2017). The validity and reliability of a 5-hz GPS device for quantifying athletes' sprints and movement demands specific to team sports.

Murr, D., Raabe, J., \& Höner, O. (2018). The prognostic value of physiological and physical characteristics in youth soccer: A systematic review. European Journal of Sport Science, 18(1), $62-74$

Newell, K. M. (1986). Constraints on the development of coordination. Motor development in children: Aspects of Coordination and Control, 34, 341-360.

Nicolairé, A. H., Cattuzzo, M. T., \& De Mello Monteiro, C. B. (2013). Questionable use of performance testing to predict success in soccer players: comment on Ljach, et al. (2012). Perceptual and Motor Skills, 116(1), 352-354.

Nicolairé, A. H., Cattuzzo, M. T., Santos Henrique, R., \& Stodden, D.F. (2016). Physical characteristics that predict involvement with the ball in recreational youth soccer. Journal of Sports Sciences, 34(18), 1716-1722.

Núñez, F. J., Toscano-Bendala, F. J., Suarez-Arrones, L., MartínezCabrera, F. I., \& De Hoyo, M. (2019). Umbrales individualizados para analizar las demandas en la aceleración en futbolistas usando GPS). Retos: Nuevas Tendencias en Educación Física, Deporte y Recreación, 35, 75-79.

Pascual, N., Orbea, J. A., Martinez, J. A., \& Jove, M. A. (2015). Analysis of physical and physiological requirements in soccer trainings in young soccer players (under -10 years). A review. Journal of Human \& Sport Exercise, 10(2), 592-601. doi:10.14198/jhse.2015.102.06.

O'Donoghue, P. (2013). Statistics for sport and exercise studies: an introduction. New York: Routledge.

O'Donoghue, P. (2015). An introduction to performance analysis of sport. London and New York: Routledge Taylor \& Francis Group.

Pankhurst, A., \& Collins, D. (2013). Talent identification and development: The need for coherence between research, system, and process. Quest, 65(1), 83-97.

Passos, P., Araújo, D., \& Volossovitch, A. (2016). Performance analysis in team sports. London and New York: Routledge Taylor \& Francis Group.

Pastor-Vicedo, J. C., González-Víllora, S., Serra-Olivares, J., \& Hernández-Martínez. Monitorización físico-táctica de futbolistas juveniles mediante el dispositivo WIMU. I Congreso Internacional en Optimización del Entrenamiento y Readaptación Físico-Deportiva Sevilla

Pazo Haro, C. I., Sáenz-López Buñuel, P., Fradua Uriondo, L., Barata Figueiredo, A. J., \& João Coelho, M. (2011). The Training of High-Level Competition Football Players from the Perspective of Academy. Apunts. Educació Física i Esports, 104, 56-65.

Pinder, R. A., Davids, K., Renshaw, I., \& Araújo, D. (2011). Representative learning design and functionality of research and practice in sport. Journal of Sport and Exercise Psychology, 33(1), 146-155.
Práxedes, A., Moreno, A., Gil-Arias, A., Claver, F., \& Del Villar, F. (2018). The effect of small-sided games with different levels of opposition on the tactical behaviour of young footballers with different levels of sport expertise. PloS one, 13(1), e0190157.

Prieto-Ayuso, A., Pastor-Vicedo, J. C., \& Contreras-Jordán, O. (2017). Content validity and psychometric properties of the nomination scale for identifying football talent (NSIFT): Application to coaches, parents and players. Sports, 5(1), 2.

Rábano-Muñoz, A., Asian-Clemente, J., Sáez de Villarreal, E., Nayler, J., \& Requena, B. (2019). Age-Related Differences in the Physical and Physiological Demands during Small-Sided Games with Floaters. Sports, 7(4), 79

Randers, M. B., Andersen, T. B., Rasmussen, L. S., Larsen, M. N., \& Krustrup, P. (2014). Effect of game format on heart rate, activity profile, and player involvement in elite and recreational youth players. Scandinavian Journal of Medicine \& Science in Sports, 24, 17-26. doi:10.1111/sms.12255

Rebelo, A., Brito, J., Seabra, A., Oliveira, J., \& Krustrup, P. (2014). Physical match performance of youth football players in relation to physical capacity. European Journal of Sport Science, 14(sup1), S148-S156. doi:10.1080/17461391.2012.664171

Renzulli, J. S., \& Gaesser, A. H. (2015). A multicriteria system for the identification of high achieving and Creative/Productive giftedness. Revista de Educación, 368, 96-131.

Rodriguez, A. N., Montenegro, O., \& Petro, J. L. Perfil dermatoglífico y somatotipificación de jugadores adolescentes de fútbol. Retos: Nuevas tendencias en educación física, deporte y recreación, 36, 32-36.

Sánchez-Sánchez, J., García, M. S., Asián-Clemente, J. A., Nakamura, F. Y., \& Ramírez-Campillo, R. (In press). Effects of the Directionality and the Order of Presentation Within the Session on the Physical Demands of Small-Sided Games in Youth Soccer. Asian Journal of Sports Medicine.

Serpiello, F. R., Duthie, G. M., Moran, C., Kovacevic, D., Selimi, E., \& Varley, M. C. (2018). The Occurrence of Repeated High Acceleration Ability (RHAA) in Elite Youth Football. International Journal of Sports Medicine, 39(07), 502-507.

Serra-Olivares, J., Clemente, F. M., \& González-Víllora, S. (2016). Tactical expertise assessment in youth football using representative tasks. SpringerPlus, 5(1), 1301.

Starkes, J. L., y Ericsson, K. A. (2003). Expert performance in sports: Advances in research on sport expertise. Champaign, IL: Human Kinetics.

Tiedemann, T., Francksen, T., \& Latacz-Lohmann, U. (2011). Assessing the performance of German Bundesliga football players: a non-parametric metafrontier approach. Central European Journal of Operations Research, 19(4), 571-587.

Travassos, B., Duarte, R., Vilar, L., Davids, K., \& Araújo, D. (2012). Practice task design in team sports: Representativeness enhanced by increasing opportunities for action. Journal of Sports Sciences, 30(13), 1447-1454.

Trecroci, A., Milanoviæ, Z., Frontini, M., Iaia, F. M., y Alberti, G. (2018). Physical performance comparison between under 15 elite and sub-elite soccer players. Journal of human kinetics, 61(1), 209-216.

Williams, A. M., \& Reilly, T. (2000). Talent Identification and Development in Soccer. Journal of Sport Sciences, 18, 657667.

Woods, C. T., Raynor, A. J., Bruce, L., \& McDonald, Z. (2016). Discriminating talent-identified junior Australian football players using a video decision-making task. Journal of sports sciences, 34(4), 342-347. 